MATHEMATICS OF COMPUTATION

Volume 70, Number 233, Pages 121-134

S 0025-5718(00)01224-2

Article electronically published on February 23, 2000

\title{
CONVERGENCE OF NUMERICAL SCHEMES FOR THE SOLUTION OF PARABOLIC STOCHASTIC PARTIAL DIFFERENTIAL EQUATIONS
}

\author{
A. M. DAVIE AND J. G. GAINES
}

\begin{abstract}
We consider the numerical solution of the stochastic partial differential equation $\partial u / \partial t=\partial^{2} u / \partial x^{2}+\sigma(u) \dot{W}(x, t)$, where $\dot{W}$ is space-time white noise, using finite differences. For this equation Gyöngy has obtained an estimate of the rate of convergence for a simple scheme, based on integrals of $\dot{W}$ over a rectangular grid. We investigate the extent to which this order of convergence can be improved, and find that better approximations are possible for the case of additive noise $(\sigma(u)=1)$ if we wish to estimate space averages of the solution rather than pointwise estimates, or if we are permitted to generate other functionals of the noise. But for multiplicative noise $(\sigma(u)=u)$ we show that no such improvements are possible.
\end{abstract}

\section{INTRODUCTION}

This paper is concerned with the convergence of numerical approximations to solutions of the stochastic partial differential equation

$$
\dot{u}=\frac{\partial^{2} u}{\partial x^{2}}+\sigma(u) \dot{W}(x t),
$$

where $\dot{W}$ is two-dimensional white noise. We assume an initial condition $u(x, 0)=$ $u_{0}(x)$ and periodic boundary conditions on $[0,1]$; the results can readily be modified to cover other self-adjoint boundary conditions.

Numerical experiments indicating convergence of finite-difference approximations to solutions of (11) were reported by Gaines [1]. A proof of convergence for a simple approximation scheme (see (2) below), and an estimate of the order of convergence, was given by Gyöngy [3]; his results in fact apply to the more general equation

$$
\dot{u}=\frac{\partial^{2} u}{\partial x^{2}}+f(x, t, u)+\sigma(x, t, u) \dot{W}(x, t) .
$$

The purpose of the present paper is to investigate to what extent these results are best possible.

We consider finite-difference approximations to (1). The simplest such approximation is the explicit scheme

$$
u_{j, m+1}=u_{j, m}+n^{2} h\left(u_{j+1, m}+u_{j-1, m}-2 u_{j, m}\right)+n \sigma\left(u_{j, m}\right) W_{j, m}
$$

Received by the editor January 6, 1999

2000 Mathematics Subject Classification. Primary 60H15, 60H35, 65M06. 
for $j=0,1, \ldots, n-1$, with $u_{-1, m}=u_{n-1, m}$ and $u_{n, m}=u_{0, m}$, and initial conditions $u_{j, 0}=u_{0}(j / n)$. Here $u_{j, m}$ is intended as an approximation to $u\left(n^{-1} j, m h\right) ; W_{j, m}=$ $W\left(R_{j, m}\right)$, where $R_{j, m}$ is the rectangle $j / n \leq x \leq(j+1) / n, m h \leq t \leq(m+1) h$. We may refer to the above scheme as the Euler scheme, since it can be obtained as the Euler approximation to a system of stochastic (ordinary) differential equations obtained by space discretisation of (1).

For the heat equation without noise, the analogue of (2) is known to be stable if $2 n^{2} h \leq 1$ and if this condition is satisfied the scheme approximates the true solution with an error of order $n^{-2}$. It is also known that better performance can be obtained using implicit methods, which allow larger time steps to be used without instability. For example, the Crank-Nicolson implicit scheme

$$
\begin{aligned}
u_{j, m+1}= & u_{j, m}+n^{2} h\left(u_{j+1, m}+u_{j-1, m}-2 u_{j, m}\right) / 2 \\
& +n^{2} h\left(u_{j+1, m+1}+u_{j-1, m+1}-2 u_{j, m+1}\right) / 2
\end{aligned}
$$

gives an error of order $n^{-2}$ with $h$ comparable with $n^{-1}$. For the equation with noise the results of [3] show that (2) gives an approximation with error of order $n^{-1 / 2}$, provided $n^{2} h \leq b<\frac{1}{2}$; we shall show that this order of approximation is best possible for schemes of this nature. The main reason why higher order methods do not give improvements is the lack of smoothness of the solution of the equation (1).

In Section 2 the special case of additive noise is considered, and some approaches are discussed for improving the order of convergence. In Section 3 it is shown that in the general case of multiplicative noise no improvement is possible using a wide class of schemes (namely those using only linear observations of the noise) which includes those considered in Section 2 In the final section we illustrate these results with some numerical examples.

We conclude this section with a discussion of known results on existence of solutions and convergence of numerical approximations. Consider the stochastic partial differential equation (1) on the region $0 \leq x \leq 1, t \geq 0$, with periodic boundary condition $u(0, t)=u(1, t)$, and initial condition $u(x, 0)=u_{0}(x) . d W(x, t)$ denotes 2-dimensional white noise. We can identify $x=0$ with $x=1$ and regard the domain as a semi-infinite cylinder. $\sigma$ is a real-valued function on $\mathbb{R}$ which is assumed to satisfy a global Lipschitz condition $|\sigma(x)-\sigma(y)| \leq L|x-y|$.

We denote by $\Gamma$ the circle formed from $[0,1]$ by identifying 0 with 1 . Addition on $\Gamma$ is $\bmod 1$.

The integral formulation of (1) is

$$
u(x, t)=\int_{\Gamma} u_{0}(y) p(x-y, t) d y+\int_{0}^{t} \int_{\Gamma} \sigma(u(y, s)) p(x-y, t-s) d W(y, s),
$$

where

$$
p(x, t)=\frac{1}{2 \sqrt{\pi t}} \sum_{m=-\infty}^{\infty} e^{-\frac{(x-m)^{2}}{4 t}}=\sum_{r=-\infty}^{\infty} e^{-4 \pi^{2} r^{2} t} e^{2 \pi i r x} .
$$

Then it is known that there exists a unique solution to (1), given $u_{0} \in L^{\infty}(\Gamma)$. The following convergence result for the scheme (2) is proved in [3]:

Theorem 1.1. Let $p \geq 2$, and suppose $u_{0}$ is Holder continuous with exponent $\frac{1}{2}$. If the number of space steps $n$ and the time step $h$ satisfy $n^{2} h \leq b$, where $0<b<\frac{1}{2}$, then

$$
\mathbb{E}\left|u_{j, m}-u(j / n, m h)\right|^{p} \leq K n^{-p / 2}
$$


for all $0 \leq j \leq n-1$ and $m$ such that $0 \leq m h \leq T$, where the constant $K$ depends only on $L, \sigma(0), u_{0}, T$ and $b$.

Remarks. 1. The proof also applies to other approximation schemes such as the Crank-Nicolson scheme.

2. It is a feature of this method that the local error is of the same order as the global error - after a single time step we already have an error of order $n^{-1 / 2}$. The reason why the local errors do not accumulate to produce a larger global error is that the main contribution to the local error consists of rapidly oscillating terms which become attenuated when propagated in time, owing to the smoothing effect of the heat equation.

From Theorem 1.1 it is straightforward to deduce a result on almost sure uniform convergence. We now consider a sequence of approximations $u_{j m}^{(k)}$ obtained using the scheme (2) with $n_{k}$ space steps and time step $h_{k}$ with $a \leq n_{k}^{2} h \leq b$. We suppose the sequence $\left\{n_{k}\right\}$ is strictly increasing. Then we have

Corollary 1.2. For any $\epsilon>0$, we have, with probability 1 , that there exists $k_{0}$ such that

$$
\sup \left|u_{j m}^{(k)}-u\left(j / n_{k}, m h_{k}\right)\right| \leq n^{\epsilon-1 / 2}
$$

for all $k>k_{0}$, where the $\sup$ is over all $0 \leq j<n$ and $m$ such that $0 \leq m h_{k} \leq T$.

Using the above corollary, it is now possible to weaken the global Lipschitz requirement on $\sigma$ to a local Lipschitz condition. More precisely, suppose $\sigma$ is locally Lipschitz on an open interval $J$ (which may be infinite); we consider the equation (1) with $u_{0}(x) \in J$. Then a solution will exist for $0 \leq t<\tau$, where $\tau$ is the first exit time from $J$. We consider a sequence of approximations $u_{j m}^{(k)}$ as in the corollary. Then one can show by standard arguments that, with probability 1 , given $\epsilon>0$ and $T<\tau$, there exists $k_{0}$ such that

$$
\sup \left|u_{j m}^{(k)}-u\left(j / n_{k}, m h_{k}\right)\right| \leq n^{\epsilon-1 / 2}
$$

for all $k>k_{0}$, where the sup is over all $0 \leq j<n$ and $m$ such that $0 \leq m h_{k} \leq T$.

The results just described indicate that solutions of equation (1) can be approximated using scheme (2) to within an average error of order $\epsilon$ using computational effort of order $\epsilon^{-6}$ (since we use $O\left(n^{3}\right)$ rectangles and the error is $O\left(n^{-1 / 2}\right)$ ). In the following sections we investigate the extent to which this performance can be improved.

\section{Additive noise: An example}

Now consider the equation

$$
\dot{u}=\frac{\partial^{2} u}{\partial x^{2}}+\dot{W}(x, t)
$$

with the initial condition $u(x, 0)=0$. We shall discuss a number of aspects of the numerical solution of this equation. First, we show that in one sense the order of approximation obtained in Theorem 1.1] cannot be improved. 
2.1. Bound on order of convergence. We fix $x$ and consider approximating $u(x, 1)$. We divide the space interval $[0,1]$ into $n$ equal steps and the time interval $[0,1]$ into $N$ equal steps. We show that, given the $W_{j, m}$ 's associated with the $N n$ rectangles of this grid, the best estimate of $u(x, 1)$ has error of order $\max \left(n^{-1 / 2}, N^{-1 / 4}\right)$.

With $h=1 / N$, we define $R_{j, m}$ and $W_{j, n}$ as before. Then we have

$$
\begin{aligned}
u(x, 1) & =\int_{0}^{1} \int_{\Gamma} p(x-y, 1-t) d W(y, t) \\
& =\sum_{j=0}^{n-1} \sum_{m=0}^{N-1} \iint_{R_{j, m}} p(x-y, 1-t) d W(y, t) .
\end{aligned}
$$

We write $\mathbb{E}_{c}$ and $\operatorname{Var}_{c}$ to denote expectation and variance conditional on knowing the $W_{j, m}$ 's. Then

$$
\mathbb{E}_{c} u(x, 1)=\sum_{j, m} p_{j, m} W_{j, m},
$$

where $p_{j, m}=n N \iint_{R_{j, m}} p(x-y, 1-t) d y d t$. Then we have

$$
\operatorname{Var}_{c} u(x, 1)=\sum_{j, m} \iint_{R_{j, m}}\left\{p(x-y, 1-t)-p_{j, m}\right\}^{2} d y d t
$$

which by symmetry we can also write as

$$
\sum_{j, m} \iint_{R_{j, m}}\left\{p(x-y, t)-q_{j, m}\right\}^{2} d y d t
$$

where $q_{j, m}=n N \iint_{R_{j, m}} p(x-y, t) d y d t$. Then we have

$$
\operatorname{Var}_{c} u(x, 1) \geq \sum_{m=0}^{N-1} \int_{m / N}^{(m+1) / N} \int_{\Gamma}\left\{p(x-y, t)-p_{m}(x-y)\right\}^{2} d y d t
$$

where $p_{m}(x-y)=N \int_{m / N}^{(m+1) / N} p(x-y, t) d t$. Expanding $p(x-y, t)$ as a Fourier series, we find that the last double integral can be written as

$$
N^{-1} \sum_{r=-\infty}^{\infty} e^{-8 \pi^{2} r^{2} m / N}\left\{\frac{1-e^{-2 \alpha}}{2 \alpha}-\frac{\left(1-e^{-\alpha}\right)^{2}}{\alpha^{2}}\right\}
$$

where $\alpha=4 \pi^{2} r^{2} / N$. The expression in curly brackets is $\geq c \min \left(\alpha^{-1}, \alpha\right)$, where $c$ is an absolute constant. Hence we obtain the lower bound

$$
\begin{aligned}
\operatorname{Var}_{c} u(x, 1) & \geq 4 \pi^{2} c N^{-2} \sum_{|r|<N^{1 / 2} /(2 \pi)} r^{2} \sum_{m} e^{-8 \pi^{2} r^{2} m / N} \\
& \geq c_{1} N^{-1 / 2},
\end{aligned}
$$

where $c_{1}$ is another absolute constant.

A similar argument, using averages with respect to $x$ instead of $t$, shows that $\operatorname{Var}_{c} u(x, 1) \geq c_{2} n^{-1}$, and it follows that the standard error of any estimate of $u(x, 1)$ based only on the $W_{j, m}$ must be at least a constant times $\max \left(n^{-1 / 2}, N^{-1 / 4}\right)$. This indicates that, using methods based on $W_{j, m}$ 's obtained from a partition into equal rectangles, one cannot improve on the order of accuracy obtained with $N=4 n^{2}$ and using the simple explicit scheme as above. 
2.2. Approximation of averages. The situation is different, at least for the case of additive noise, if instead of estimating $u(x, t)$ pointwise one wishes to estimate a space average, such as $(b-a)^{-1} \int_{a}^{b} u(x, t) d x$ or more generally $\int_{\Gamma} \phi(x) u(x, t) d x$, where $\phi$ is some given weight function with $\int_{\Gamma} \phi=1$. We show that, in the case of the equation $\dot{u}=\partial^{2} u / \partial x^{2}+\dot{W}$, we get more accurate approximations for such averages than for pointwise estimates, and moreover taking $h$ to be of order $n^{-2}$ is no longer optimal.

Assuming zero initial condition for simplicity, the solution can be written

$$
u(x, t)=\int_{0}^{t} \int_{\Gamma} p(x-y, t-s) d W(y, s)
$$

we wish to estimate the quantity

$$
X_{t}=\int_{\Gamma} u(x, t) \phi(x) d x=\int_{\Gamma} \int_{0}^{t} \int_{\Gamma} \phi(x) p(x-y, t-s) d W(y, s) d x
$$

Expanding $\phi$ as a Fourier series $\phi(x)=\sum c_{r} e^{-2 \pi i r x}$, we obtain

$$
X_{t}=\sum_{r} \int_{0}^{t} \int_{\Gamma} c_{r} e^{-4 \pi^{2} r^{2}(t-s)} e^{-2 \pi i r y} d W(y, s) .
$$

It will turn out that it is optimal to use a value of $h$ comparable to $n^{-1}$, so that to avoid instability we need to use a scheme such as the Crank-Nicolson scheme

$$
\begin{aligned}
u_{j, m+1}= & u_{j, m}+n^{2} h\left(u_{j+1, m}+u_{j-1, m}-2 u_{j, m}\right) / 2 \\
& +n^{2} h\left(u_{j+1, m+1}+u_{j-1, m+1}-2 u_{j, m+1}\right) / 2+n W_{j, m}
\end{aligned}
$$

or the implicit scheme (often called the backward Euler scheme)

$$
u_{j, m+1}=u_{j, m}+n^{2} h\left(u_{j+1, m+1}+u_{j-1, m+1}-2 u_{j, m+1}\right)+n W_{j, m} .
$$

We carry out the analysis for the scheme (5); for (41) the analysis is similar.

Using vector notation we can write the scheme (5), as applied to the present equation, as

$$
\left(I-n^{2} h A\right) u_{m+1}=u_{m}+n W_{m},
$$

where $u_{m}$ is the column vector with entries $u_{0, m}, \cdots, u_{n-1, m}$, and $W_{m}$ similarly; $\sigma\left(u_{0, m}\right)$ is the diagonal matrix with entries $\sigma\left(u_{0, m}\right), \cdots, \sigma\left(u_{n-1, m}\right)$, and $A$ is the matrix

$$
A=\left[\begin{array}{rrrrrr}
-2 & 1 & 0 & \ldots & 0 & 1 \\
1 & -2 & 1 & \ldots & 0 & 0 \\
0 & 1 & -2 & \ldots & 0 & 0 \\
& \ldots & & \ldots & & \\
0 & 0 & 0 & \ldots & -2 & 1 \\
1 & 0 & 0 & \ldots & 1 & -2
\end{array}\right]
$$

With $u_{0}=0$ we deduce that

$$
u_{m}=n\left(B^{m} W_{0}+B^{m-1} W_{1}+\cdots+B W_{m-1}\right),
$$

where $B=\left(I-n^{2} h A\right)^{-1}$.

Now $A$ has eigenvalues $-4 \sin ^{2}(\pi r / n), r=0, \cdots, n-1$, with corresponding eigenvector $\psi_{r}$ having components $\psi_{r, j}=e^{2 \pi i r j / n}$. Using these to diagonalise $A$, we find that the matrix $B^{k}$ has entries given by $\left[B^{k}\right]_{j l}=n^{-1} \sum_{r=0}^{n-1} e^{2 \pi i r(j-l) / n} \mu_{r}^{k}$, where $\mu_{r}=\left(1+4 n^{2} h \sin ^{2}(\pi r / n)\right)^{-1}$. 
We introduce the notation $\sum_{r}^{\prime}$ for $\sum_{|r|<n / 2}$ if $n$ is odd and $\sum_{r=1-n / 2}^{n / 2}$ if $n$ is even, and $\sum_{r}^{\prime \prime}$ for a sum over all $r$ not included in $\sum^{\prime}$. Then the approximate solution $u_{j, m}$ is given by

$$
u_{j, m}=\sum_{k=0}^{m-1} \sum_{l=0}^{n-1} \sum_{r}^{\prime} e^{2 \pi i r(j-l) / n} \mu_{r}^{m-k} W_{l, k},
$$

and we approximate $X_{t}$ for $t=k / m$ by $\tilde{X}_{k}=n^{-1} \sum_{j=0}^{n-1} \phi(j / n) u_{j, m}$. Writing $\tilde{c}_{r}=\sum_{P=-\infty}^{\infty} c_{r+P n}$, we find by expanding $\phi$ as a Fourier series that

$$
\begin{aligned}
\tilde{X}_{k} & =\sum_{k=0}^{m-1} \sum_{r}^{\prime} \sum_{l=0}^{n-1} \tilde{c}_{r} e^{-2 \pi i r l / n} \mu_{r}^{m-k} W_{l, k} \\
& =\sum_{k=0}^{m-1} \sum_{r}^{\prime} \tilde{c}_{r} \mu_{r}^{m-k} \int_{k h}^{(k+1) h} \int_{\Gamma} e^{-2 \pi i r l(x) / n} d W(x, t),
\end{aligned}
$$

where $l(x)$ is the integer $l$ such that $\frac{l}{n} \leq x<\frac{l+1}{n}$. We can expand $e^{-2 \pi i r l(x) / n}$ as a Fourier series $\sum_{P=-\infty}^{\infty} \delta_{r+P n} e^{-2 \pi i(r+P n) x}$, where

$$
\delta_{r}= \begin{cases}\left(e^{2 \pi i r / n}-1\right) \frac{n}{2 \pi i r}, & r \neq 0 \\ 1, & r=0\end{cases}
$$

and then we obtain $\tilde{X}_{k}=\sum_{k=0}^{m-1} \sum_{r=-\infty}^{\infty} \tilde{c}_{r} \mu_{r}^{m-k} \delta_{r} \int_{k h}^{(k+1) h} \int_{\Gamma} e^{-2 \pi i r x} d W(x, t)$. We have then

$$
\mathbb{E}\left|X_{t}-\tilde{X}_{k}\right|^{2}=\sum_{k=0}^{m-1} \sum_{r=-\infty}^{\infty} \int_{k h}^{(k+1) h}\left|c_{r} e^{-4 \pi^{2} r^{2}(t-s)}-\tilde{c}_{r} \mu_{r}^{m-k} \delta_{r}\right|^{2} d s .
$$

Now we can prove:

Theorem 2.1. Suppose $\phi$ is absolutely continuous with $L^{2}$ derivative. Then, for either of the schemes (4) and (5) we have $\mathbb{E}\left(X_{k / m}-\tilde{X}_{k}\right)^{2}=O\left(\max \left(n^{-2}, h^{2}\right)\right)$.

Proof. The hypothesis on $\phi$ is equivalent to $\sum r^{2}\left|c_{r}\right|^{2}<\infty$. From this it follows that $\sum_{r}^{\prime}\left|c_{r}-\tilde{c}_{r}\right|^{2} \leq \sum_{r}^{\prime}\left(\sum_{P \neq 0}(r+n P)^{2}\left|c_{r+n P}\right|^{2}\right)\left(\sum_{P \neq 0}(r+n P)^{-1}\right) \leq$ $\pi^{2} n^{-2} \sum r^{2}\left|c_{r}\right|^{2}=O\left(n^{-2}\right)$, and hence $\sum_{r}^{\prime} r^{2} n^{-2}\left|\tilde{c}_{r}\right|^{2}=O\left(n^{-2}\right)$. Also, we find that $\left|\delta_{r+P n}\right|=O\left(P^{-1} n^{-1}|r|\right)$ if $P \neq 0$, and so

$$
\sum_{r}^{\prime \prime}\left|\tilde{c}_{r} \delta_{r}\right|^{2}=O\left(\sum_{r}^{\prime} \sum_{|P|>0}\left|\tilde{c}_{r}\right|^{2} r^{2} n^{-2} P^{-2}\right)=O\left(\sum^{\prime} r^{2} n^{-2}\left|\tilde{c}_{r}\right|^{2}\right)=O\left(n^{-2}\right)
$$

In addition we have $1-\delta_{r}=O(r / n)$, and so $\sum^{\prime}\left|\tilde{c}_{r}\right|^{2}\left|1-\delta_{r}\right|^{2}=O\left(n^{-2}\right)$.

Moreover we have $\sum_{r}^{\prime \prime}\left|c_{r}\right|^{2} \int_{0}^{t} e^{-8 \pi^{2} r^{2}(t-s)} d s=O\left(n^{-2}\right)$. Noting that $0<\mu_{r} \leq 1$, we see from the above estimates that we only have to show that

$$
\sum_{k=1}^{m-1} \sum_{r}^{\prime} \int_{k h}^{(k+1) h}\left|c_{r}\right|^{2}\left\{e^{-4 \pi^{2} r^{2}(t-s)}-\mu_{r}^{m-k-1}\right\}^{2} d s=O\left(n^{-2}+h^{2}\right) .
$$

To do this we note that if $8 \pi h r^{2} \leq 1$ then $\log \mu_{r}=-4 \pi^{2} r^{2} h+O\left(h r^{4} n^{-2}+h^{2} r^{4}\right)$, and so $\log \mu_{r}^{Q}=-4 \pi^{2} r^{2}(t-s)+O\left(r^{2} h+Q h r^{4} n^{-2}+Q h^{2} r^{4}\right)$, where $Q=m-k$. 
Thus $\mu_{r}^{Q}-e^{-4 \pi^{2} r^{2}(t-s)}=O\left(r^{2} h+Q h r^{4} n^{-2}+Q h^{2} r^{4}\right) e^{-c Q r^{2} h}$. From this it follows that for such $r$

$$
\sum_{k=1}^{m-1} \int_{k h}^{(k+1) h}\left\{e^{-4 \pi^{2} r^{2}(t-s)}-\mu_{r}^{Q}\right\}^{2} d s=O\left(r^{2}\left(h^{2}+n^{-4}\right)\right) .
$$

Next, for $r$ satisfying $8 \pi h r^{2}>1$ but $|r| \leq n / 2$ we have $\mu_{r}^{Q} \leq e^{-c Q h /\left(h r^{2}\right)}$ for either choice of $\mu_{r}$, and so $\sum_{k} \mu_{r}^{2 Q}=O\left(h r^{2}\right)$; since $\int_{0}^{t} e^{-8 \pi^{2} r^{2}(t-s)} d s=O(h)$ we conclude that (6) holds also for such $r$, and hence for all $r$ with $|r| \leq n / 2$. Multiplying by $\left|a_{r}\right|^{2}$ and summing over $r$ then gives the desired conclusion.

A similar analysis applies to the scheme (4), and we get the same result for this scheme.

So if we take $h$ to be comparable to $n^{-1}$, and use one of the two schemes mentioned above, we obtain an error of order $n^{-1}$ with $O\left(n^{2}\right)$ rectangles, which is a great improvement on the order obtained for pointwise estimates.

For averages of the form $\int_{a}^{b} u(x, t) d t$, in which case $\phi$ is the characteristic function of an interval, which does not satisfy the requirement of Theorem 2.1, we get a somewhat poorer estimate - calculations similar to those used in the proof of Theorem 2.1 show that, in the case of scheme (5), the error is of order $\max \left(n^{-1}, h^{3 / 4}\right)$, and in the case of scheme (4) it is slightly worse still, of order $\max \left(n^{-1}, h n^{1 / 2}\right)$.

2.3. Exact generation of solutions of equation (3). The solution of (3) has a Gaussian distribution, a fact which enables us to generate values of $u(x, t)$ at any given finite set of $(x, t)$ points. We now show how this can be done efficiently for a rectangular grid of points of the type we have been considering. With $h$ and $n$ as before, we let $X_{j, m}=u\left(\frac{j}{n}, m h\right)$ and consider the problem of generating the $X_{j, m}$. For convenience we suppose $n$ is even. We shall encounter terms bounded by $e^{-\pi^{2} n^{2} h}$; under mild assumptions on $n$ and $h$ such terms are negligible, and we shall assume this is the case. For example, if $n^{2} h \geq 4$ then $e^{-\pi^{2} n^{2} h}<10^{-17}$. We use the symbol $\approx$ for approximations obtained by neglecting such terms.

For the solution $u$ we have the expression

$$
\begin{aligned}
u(x, t) & =\int_{0}^{t} \int_{\Gamma} \sum_{r=-\infty}^{\infty} e^{-4 \pi^{3} r^{2}(t-s)} e^{2 \pi i r(x-y)} d W(s, y) \\
& =\sum_{r} e^{2 \pi i r x} \int_{0}^{t} e^{-4 \pi^{2} r^{2}(t-s)} d W_{r}(s),
\end{aligned}
$$

where $d W_{r}(s)=\int_{\Gamma} e^{-2 \pi i r y} d W(y, s)$. Using this, we can write $X_{j, m}$ as a finite Fourier transform: $X_{j, m}=\sum_{k}^{\prime} x_{k, m} e^{2 \pi i j k / N}$, where

$$
x_{k, m}=\sum_{P=-\infty}^{\infty} \int_{0}^{m h} e^{-4 \pi^{2}(k+n P)^{2}(m h-s)} d W_{k+n P}(s) .
$$

The $W_{r}$ are complex Brownian motions satisfying

$$
W_{r}(s)=\bar{W}_{-r}(s) \quad \text { and } \quad \mathbb{E} W_{r}(s) W_{-q}(t)=\delta_{q r} \min (s, t)
$$

so that $W_{r}$ and $W_{q}$ are independent unless $r=q$ or $-q$. Note that $x_{-k, m}=\bar{x}_{k, m}$ (which ensures that $X_{k, m}$ is real). Thus it suffices to generate $x_{k, m}$ for $0 \leq k \leq n / 2$. 
For $0 \leq k<\frac{n}{2}$ it is convenient to write $x_{k, m}=y_{k, m}+z_{k, m}$, where

$$
y_{k, m}=\int_{0}^{m h} e^{-4 \pi^{2} k^{2}(m h-s)} d W_{k}(s)
$$

and

$$
\begin{aligned}
z_{k, m} & =\sum_{P \neq 0} \int_{0}^{m h} e^{-4 \pi^{2}(k+n P)^{2}(m h-s)} d W_{k+n P}(s) \\
& \approx \sum_{P \neq 0} \int_{(m-1) h}^{m h} e^{-4 \pi^{2}(k+n P)^{2}(m h-s)} d W_{k+n P}(s) .
\end{aligned}
$$

We can also write

$$
y_{k, m+1}=e^{-4 \pi^{2} k^{2} h} y_{k, m}+v_{k, m+1}
$$

where $v_{k, m}=\int_{(m-1) h}^{m h} e^{-4 \pi^{2} k^{2}(m h-s)} d W_{k}(s)$. Then we have that the random variables $v_{k, m}\left(0 \leq k<\frac{n}{2}\right), z_{k, m}$ and $x_{n / 2, m}$ are effectively independent (any correlations being bounded by $\left.e^{-\pi^{2} n^{2} h}\right)$, and we have

$$
\begin{aligned}
\mathbb{E}\left|v_{0, m}\right|^{2}=h, & \mathbb{E}\left|v_{k, m}\right|^{2}=\frac{1-e^{-8 \pi^{2} k^{2} h}}{8 \pi^{2} k^{2}} \quad \text { for } 0<k<\frac{n}{2}, \\
\mathbb{E}\left|z_{k, m}\right|^{2} & =\sum_{P \neq 0} \frac{1-e^{-8 \pi^{2}(k+n P)^{2} h}}{8 \pi^{2}(k+n P)^{2}} \sum_{P \neq 0} \frac{1}{8 \pi^{2}(k+n P)^{2}} \\
& =\left\{\begin{array}{cl}
\left(24 n^{2}\right)^{-1}, & k=0, \\
\frac{1}{8}\left(\frac{1}{n^{2} \sin ^{2} \frac{\pi k}{n}}-\frac{1}{\pi^{2} k^{2}}\right), & 0<k<\frac{n}{2},
\end{array}\right.
\end{aligned}
$$

and

$$
\mathbb{E}\left(x_{n / 2, m}\right)^{2} \approx \sum_{k=0}^{\infty} \frac{2}{8 \pi^{2} n^{2}\left(k+\frac{1}{2}\right)^{2}}=\left(8 n^{2}\right)^{-1} .
$$

We can now describe the procedure for generating the $x_{k, m}$. For each $m$, generate $A_{k}, B_{k}, C_{k}$ and $D_{k}$ to be independent with $N(0,1)$ distribution for $0 \leq k<n / 2$ (actually $B_{0}$ is not needed). Then set

$$
v_{k, m}= \begin{cases}h A_{0}, & k=0 \\ a_{k}\left(A_{k}+i B_{k}\right), & 0<k<n / 2,\end{cases}
$$

where

$$
a_{k}=\frac{1}{4 \pi k} \sqrt{1-e^{-8 \pi^{2} k^{2} h}}
$$

and

$$
z_{k, m}= \begin{cases}\frac{1}{n \sqrt{24}} C_{0}, & k=0 \\ b_{k}\left(C_{k}+i D_{k}\right), & 0<k<n / 2,\end{cases}
$$

where

$$
b_{k}=\frac{1}{4}\left\{\frac{1}{n^{2} \sin ^{2}(\pi k / n)}-\frac{1}{\pi^{2} k^{2}}\right\}^{1 / 2},
$$

and finally set $x_{n / 2, m}=\frac{1}{N \sqrt{8}} D_{0}$. 
Then for $0 \leq k<\frac{n}{2}$ we obtain $y_{k, m}$ inductively by $y_{k, m+1}=e^{-4 \pi^{2} k^{2} h} y_{k, m}+$ $v_{k, m+1}$ with $y_{k, 0}=0$, and then set $x_{k, m}=y_{k, m}+z_{k, m}$. Finally we obtain $X_{j, m}=$ $\sum_{k}^{\prime} x_{k, m} e^{2 \pi i j k / n}$.

If we use the fast Fourier transform for the last step, then the computational load (for unit time, i.e. $O\left(h^{-1}\right)$ time steps) is of order $h^{-1} n \log n$.

Of course, for a nonlinear equation such as (1) the solutions will not be normally distributed, and one cannot expect to generate the solution at particular points exactly in the manner just described. However it is still reasonable to hope that by generating suitable linear functionals of the noise (which will be normally distributed) one may be able to improve the order of approximation. However the example described in the next section shows that in fact none of the approaches discussed in this section can improve on the order given by Theorem 1.1 in general. We may still expect these approaches to give improvements for equations such as $\dot{u}=\frac{\partial^{2} u}{\partial x^{2}}+f(x, t, u)+\dot{W}(x, t)$, where the noise is additive but nonlinear terms are present. We hope to describe detailed numerical implementations for such equations in a future publication.

\section{Multiplicative nOise: An example}

For the case of multiplicative noise, even if it is averages which we wish to approximate, the order of convergence obtained in Theorem 1.1 cannot be improved, at least by the methods we have been considering. We shall illustrate this by considering the equation

$$
\dot{u}=\frac{\partial^{2} u}{\partial x^{2}}+u \dot{W}(x, t)
$$

with the initial condition $u(x, 0)=1$. We consider the problem of approximating $X=\int_{\Gamma} u(x, 1) d x$. Let $N$ be a positive integer and let $f_{1}, \cdots, f_{N} \in L^{2}(S)$, where $S=\Gamma \times[0,1]$. Let $X_{i}=\int_{S} f_{i}(x, t) d W(x, t)$ for $i=1, \cdots, N$. Then we have

Theorem 3.1. For any measurable function $G$ of $N$ real variables we have

$$
\mathbb{E}\left\{X-G\left(X_{1}, \cdots, X_{N}\right)\right\}^{2} \geq c N^{-1 / 3},
$$

where $c$ is a positive absolute constant.

The theorem shows that if we wish to approximate the average $\int_{\Gamma} u(x, 1) d x$ with error $\epsilon$ using linear observations of the noise, then we require a number of observations of order $\epsilon^{-6}$, which is the same as the order given by Theorem 1.1

Proof. Consider the space $Z$ of processes $v(x, t)$ such that, for each $(x, t), v(x, t)$ is measurable with respect to the $\sigma$-algebra generated by $\{W(y, s): 0 \leq s<t\}$, and satisfying $\sup _{S} \mathbb{E} v(x, t)^{2}<\infty$. On $Z$ we define the operator $T$ by $T v(x, y)=$ $\int_{0}^{t} \int_{\Gamma} p(x-y, t-s) v(y, s) d W(y, s)$. Straightforward estimates show that $T$ is a contraction with respect to the norm given by $\|v\|^{2}=\sup _{S} e^{-M t} \mathbb{E} v(x, t)^{2}$.

Now we have $u=1+T u$, and since $T$ is a contraction we have the convergent expansion $u(x, t)=\sum_{r=0}^{\infty} v^{(r)}(x, t)$, where $v^{(0)}(x, t)=1$ and $v^{(r)}=T^{r} v^{0)}$. Then we have $X=1+I_{1}+I_{2}+\cdots$, where

$$
I_{r}=\int_{\Gamma} v^{(r)}\left(x, t_{1}\right) d x=\int_{0}^{1} \int_{\Gamma} v^{(r-1)}(y, s) d W(y, s) .
$$


We have

$$
\begin{gathered}
I_{1}=\int_{S} d W(x, t), \\
I_{2}=\int_{S} \int_{S} \mathbb{I}_{s<t} p(x-y, t-s) d W(y, s) d W(x, t),
\end{gathered}
$$

where $\mathbb{I}_{A}$ denotes the indicator function of $A$, and so on.

We now make use of the Wiener 'homogeneous chaos' decomposition of $L^{2}(\mathcal{P})$, where $\mathcal{P}$ is the underlying probability measure of the white noise process on $S$,

$$
L^{2}(\mathcal{P})=C_{0} \oplus C_{1} \oplus C_{2} \oplus \cdots .
$$

The spaces $C_{0}, C_{1}$, etc., are mutually orthogonal, and $C_{0} \oplus C_{1} \oplus \cdots \oplus C_{n}$ coincides with the space of polynomial functionals of degree $\leq n$. See [4, p. 354].

We then have $I_{1} \in C_{1}, I_{2} \in C_{2}$, etc. We also have

$$
G\left(X_{1}, \cdots, X_{N}\right)=\sum_{k=0}^{\infty} G_{k}\left(X_{1}, \cdots, X_{N}\right),
$$

where $G_{k}\left(X_{1}, X_{2}, \cdots, X_{N}\right) \in C_{k}$. Then

$$
\mathbb{E}\left(X-G\left(X_{1}, \cdots, X_{N}\right)\right)^{2} \geq \mathbb{E}\left(I_{2}-G_{2}\left(X_{1}, \cdots, X_{N}\right)\right)^{2} .
$$

We also have $G_{2}\left(X_{1}, \cdots, X_{N}\right)=\sum_{j, k=1}^{N} a_{j k} X_{j} X_{k}-d$, where $A=\left(a_{j k}\right)$ is a symmetric matrix and $d=\mathbb{E} \sum_{j, k=1}^{N} a_{j k} X_{j} X_{k}$. Then $\mathbb{E}\left(I_{2}-G_{2}\left(X_{1}, \cdots, X_{N}\right)\right)^{2}$ equals

$$
\frac{1}{4} \int_{S} \int_{S}\left\{p(x-y,|t-s|)-2 \sum a_{j k} f_{j}(x, t) f_{k}(y, s)\right\}^{2} d y d s d x d t .
$$

It remains to obtain a lower bound for the above integral. In essence this results from the spectral theory of the integral operator with kernel $p(x-y,|t-s|)$; but we present the proof in a manner which does not assume any operator theory. The proof is accomplished in the following two lemmas. The first is an elementary piece of matrix algebra which is no doubt known, but we could not find a suitable reference.

Lemma 3.2. Let $N$ and $P$ be positive integers with $P>N$. Let $\Lambda$ be a diagonal $P \times P$ matrix with real entries $\lambda_{1}, \cdots, \lambda_{P}$ satisfying $\lambda_{j}^{2} \geq \delta>0$. Let $A$ be a real symmetric $N \times N$ matrix and $C$ a real $N \times P$ matrix. Then

$$
\operatorname{tr}\left(\Lambda-C^{t} A C\right)^{2} \geq(P-N) \delta
$$

Proof. By continuity it suffices to prove the result when the $\lambda_{j}$ are all different and $A$ is nonsingular; by multiplying $A$ by a constant we may also suppose $\operatorname{tr}\left(C^{t} C\right)=1$. Then we fix such $\Lambda$ and $A$ and define a function on the set of $N \times P$ matrices by $g(C)=\operatorname{tr}\left(\Lambda-C^{t} A C\right)^{2}$. We then choose $C$ to minimise $g$ over the set $S=\{C$ : $\left.\operatorname{tr}\left(C^{t} C\right)=1\right\}$, which can be thought of as the unit sphere in $\mathbb{R}^{N P}$. Then, for any $N \times P$ matrix $Y$ such that $Y^{t} C=0$, the straight line $\{C+t Y\}$ is tangent to $S$ at $C$, and so $0=\left.\frac{d}{d t} g(C+t Y)\right|_{t=0}=-4 \operatorname{tr} Y^{t} A C \Lambda+4 \operatorname{tr} Y^{t} A C C^{t} A C$. Since this holds for all $Y$ such that $Y^{t} C=0$, it follows that $-A C \Lambda+A C C^{t} A C+\mu C=0$ for some real $\mu$. Then, since $A$ is invertible, we deduce that $C \Lambda=B C$, where $B=C C^{t} A+\mu A^{-1}$. This means that if $c_{j}$ is the $j$ th column of $C$, then $\lambda_{j} c_{j}=B c_{j}$. So for each $j$, either $c_{j}=0$ or $\lambda_{j}$ is an eigenvalue of $B$. Since the $\lambda_{j}$ are distinct, the latter can occur for at most $N$ values of $j$. For the remaining values of $j$, which number at least $P-N$, we have $c_{j}=0$, and so the $j$ th diagonal entry of $\Lambda-C^{t} A C$ 
is $\lambda_{j}$, and so $g(C) \geq(P-N) \delta$. This conclusion holds when $C$ minimises $g(C)$, and hence it holds for all $C \in S$, completing the proof.

Lemma 3.3. There is a positive constant $c$ such that, if $N$ is a positive integer, $A=\left(a_{j k}\right)$ is a symmetric $N \times N$ matrix, and $f_{1}, \cdots, f_{N} \in L^{2}(S)$, then

$$
\int_{S} \int_{S}\left\{p(x-y,|t-s|)-\sum_{j, k=1}^{N} a_{j k} f_{j}(x, t) f_{k}(y, s)\right\}^{2} d y d s d x d t \geq c N^{-1 / 3}
$$

Proof. For positive integers $r, n$ let $\alpha_{r}=4 \pi^{2} r^{2}$ and let $\omega_{r n}$ be a solution of the equation

$$
\tan \omega=\frac{2 \alpha_{r} \omega}{\omega^{2}-\alpha_{r}^{2}}
$$

in the interval $\left(n-\frac{1}{2}\right) \pi<\omega<\left(n+\frac{1}{2}\right) \pi$; that such a solution must exist is clear from the graph of $\tan \omega$.

Next define $\phi_{r n}(x, t)=c_{r n} \cos (2 \pi r x)\left(\alpha_{r} \sin \omega_{r n} t-\omega_{r n} \cos \omega_{r n} t\right)$, where the constant $c_{r n}$ is chosen so that $\int_{S} \phi_{r n}^{2}=1$. Then an elementary calculation shows that

$$
\int_{S} p(x-y,|t-s|) \phi_{r n}(y, s) d y d s=\lambda_{r n} \phi_{r n}(x, t) \quad \text { where } \lambda_{r n}=\frac{2 \alpha_{r}}{\alpha_{r}^{2}+\omega_{r n}^{2}},
$$

in other words $\phi_{r n}$ is an eigenvector of the integral operator with kernel $p(x-y,|t-s|)$. Then the orthogonality of eigenvectors shows that the $\phi_{r n}$ are mutually orthogonal.

Let $M$ be the smallest integer with $M^{3}>6 N$. Let $P$ be the number of pairs $(r, n)$ of positive integers with $r \leq M$ and $n \leq r^{2}$, so that $P=\sum_{r=1}^{M} r^{2}>M^{3} / 3>2 N$. We relabel the $\phi_{r n}$ for $(r, n)$ satisfying this condition, in some order, as $\phi_{1}, \cdots, \phi_{P}$, and the same for $\lambda_{1}, \cdots, \lambda_{P}$. We then have $\lambda_{j}^{2}>$ const. $M^{-4}$ for $j=1, \cdots, P$.

Now let $c_{j l}=\int_{S} f_{j} \phi_{l}$; we see that the orthogonal projection of

$$
p(x-y,|t-s|)-\sum \alpha_{j k} f_{j}(x, t) f_{k}(y, s)
$$

on the span of the set of functions $\phi_{l}(x, t) \phi_{m}(y, s)$ in $L^{2}(S \times S)$ is

$$
\sum \lambda_{l} \phi_{l}(x, t) \phi_{l}(y, s)-\sum \alpha_{j k} c_{j l} c_{k m} \phi_{l}(x, t) \phi_{m}(y, s) .
$$

It follows that

$$
\int_{S} \int_{S}\left\{p(x-y,|t-s|)-\sum_{j, k=1}^{N} a_{j k} f_{j}(x, t) f_{k}(y, s)\right\}^{2} d y d s d x d t \geq \operatorname{tr}\left(\Lambda-C^{t} A C\right)^{2},
$$

where $\Lambda$ is the diagonal matrix with entries $\lambda_{1}, \cdots, \lambda_{P}$ and $C=\left(c_{j l}\right)$. It follows from Lemma 3.2 that $\operatorname{tr}\left(\Lambda-C^{t} A C\right)^{2} \geq$ const. $N M^{-4} \geq$ const. $N^{-1 / 3}$, which completes the proof.

The theorem now follows from Lemma 3.3 .

This result indicates that to improve on the order of convergence given by Theorem 1.1 one would have to generate integrals which are quadratic in the noise, just as one has to generate area integrals to do better than order $\frac{1}{2}$ convergence for general finite-dimensional stochastic differential equations (see [2]). The latter has only been achieved in the 2-dimensional case, which suggests that there 
are formidable obstacles to doing this in the infinite-dimensional situation under consideration here.

\section{NuMERICAL EXAMPLES}

We have generated three sets of numerical results to illustrate the findings of the paper. The first set consists of pathwise approximations of equations (3) and (7). The second set contains space averages, $\int_{\Gamma} \phi(x) u(x, t) d x$, as defined in subsection 2.2, of solutions to the same two equations. Finally, we have generated an exact solution in the case of additive noise, using the method outlined in subsection 2.3 .

4.1. Pathwise solutions. For each of equations (3) and (17), that is, in the case of additive and multiplicative noise, we have generated 100 independent sets of pathwise solutions. Each set contains approximate solutions using four different space steps with the same realisation of the Brownian sheet $W(x, t)$. The method used for generating approximate solutions using a single Brownian sheet and different time and space steps is described in [1].

We have tried three different discretisation schemes: the explicit Euler scheme, given in (2), and the two semi-implicit schemes given in (4) and (5). For each scheme, the number of space steps used is $N_{1}=16, N_{2}=32, N_{3}=64$ and $N_{4}=128$. For the Euler scheme, we take the time step $h=\frac{1}{4 N^{2}}$ (since we have to ensure stability). For the other two schemes we take $h=\frac{1}{4 N}$. In all cases the final time is $T=0.125$.

We measure the difference at the final time between the approximate solutions obtained with two different space steps, by adding the squared difference over the 16 space points which are common to all solutions and then summing over the 100 different realisations of the Brownian sheet. We define

$$
S_{i}=\sum_{j=1}^{100} \sum_{k=1}^{16}\left(u_{j, k}^{i}-u_{j, k}^{i+1}\right)^{2}
$$

for $i=1,2,3$, where by $u_{j, k}^{i}$ we denote the approximation to $u\left(x_{k}, T\right)$ obtained using $N=N_{i}$ and using the $j$ th independent realisation of the Brownian sheet. The space points are $x_{k}=k / N_{1}$. The order of convergence can be seen by considering the ratios $S_{1} / S_{2}$ and $S_{2} / S_{3}$.

The results are presented in Table 1 The first three rows are the results for additive noise and the second three for multiplicative noise. In both cases, we see that, as expected, when we have $h=\frac{1}{4 N^{2}}$, the values of $S_{1} / S_{2}$ and $S_{2} / S_{3}$ are close to 2 , and when $h=\frac{1}{4 N}$ the values are close to $\sqrt{2}$, confirming that there is no gain in order of convergence obtainable by using the schemes (4) and (5) rather than the simple Euler scheme (2). However, the values of the approximation errors, given by $S_{1}, S_{2}$ and $S_{3}$ in this table, indicate that the leading coefficient of the error must be much smaller in the case of the semi-implicit schemes. Therefore, when we take into account the much larger time-step used with the semi-implicit schemes, it seems clearly advantageous to use them instead of scheme (2), and also the scheme (5) gives considerably smaller errors than the scheme (4).

We remark that for deterministic problems, (4), being second-order, performs better than (5). However this does not apply to the problem considered here; because of the lack of smoothness of the solution, a method which is second-order in the deterministic case need not perform better than one which is first-order. 
TABLE 1. Pathwise solutions

\begin{tabular}{|c|c|c|c|c|c|c|}
\hline Equation & Scheme & $S_{1}$ & $S_{2}$ & $S_{3}$ & $S_{1} / S_{2}$ & $S_{2} / S_{3}$ \\
\hline (3) & (2) & 15.14 & 7.14 & 3.63 & 2.12 & 1.97 \\
\hline (3) & (4) & 30.03 & 21.84 & 14.84 & 1.38 & 1.47 \\
\hline (3) & (5) & 4.64 & 2.98 & 1.77 & 1.57 & 1.55 \\
\hline (77) & (2) & 20.98 & 10.15 & 5.40 & 2.07 & 1.88 \\
\hline (7) & (4) & 19.23 & 14.41 & 9.72 & 1.33 & 1.48 \\
\hline (7) & (5) & 7.16 & 4.34 & 2.45 & 1.65 & 1.77 \\
\hline
\end{tabular}

4.2. Space averages. Here we illustrate the results of subsection 2.2, by approximating $\bar{u}(T)=\int_{\Gamma} \phi(x) u(x, T) d x$ for $\phi(x)=1 /\{2+\cos (2 \pi x)\}$, where $u(x, t)$ is the solution to either (3) or (7). As in the previous section, we have generated 100 sets of results, where in each set we use a single representation of the noise and produce approximations for various different numbers of space steps and three different numerical schemes. In this section, let

$$
S_{i}=\sum_{j=1}^{100}\left(\bar{u}_{j}^{i}-\bar{u}_{j}^{i+1}\right)^{2}
$$

for $i=1,2,3$, where

$$
\bar{u}_{j}^{i}=\frac{1}{N_{i}} \sum_{k=0}^{N_{i}-1} \phi\left(x_{k}^{i}\right) u_{j, k}^{i}
$$

$x_{k}^{i}=k / N_{i}$ and $u_{j, k}^{i}$ is the approximation to $u\left(x_{k}^{i}, T\right)$ obtained using $N=N_{i}$ and the $j$ th independent realisation of the Brownian sheet. $N_{i}$ takes the same values as for the pathwise results.

When producing the results given in Table 21 we have again taken $h=\frac{1}{4 N^{2}}$ for the Euler scheme (2) and $h=\frac{1}{4 N}$ for the other schemes. The table shows that with additive noise, the ratio $S_{i} / S_{i+1}$ is roughly 4 , whatever the numerical scheme used, confirming that in this case it makes sense to use one of the semi-implicit schemes, (4) or (5), and avoid the need to take very small time-steps. In the case of multiplicative noise, as proved in Section 3 we can see that the order of convergence is no better for space averages than for pathwise approximations, since the values of $S_{i} / S_{i+1}$ are similar to those in Table 1 and there is therefore not such an obvious gain in using anything other than the Euler scheme. However, here again, through a difference in leading coefficients, it would seem possible to obtain the same size error with a semi-implicit scheme as with the Euler scheme, but with the semiimplicit scheme using larger time-steps, and therefore, although this is not as clear cut as in Table 1 computing time could still be reduced by using a semi-implicit scheme.

\begin{tabular}{|c|c|c|c|c|c|c|}
\hline Equation & Scheme & $S_{1}$ & $S_{2}$ & $S_{3}$ & $S_{1} / S_{2}$ & $S_{2} / S_{3}$ \\
\hline (3) & (2) & .0013 & .00031 & .000076 & 4.22 & 4.10 \\
\hline (3) & (44) & .0018 & .00047 & .00011 & 3.78 & 4.21 \\
\hline (3) & (15) & .0016 & . 00051 & . 00011 & 3.20 & 4.85 \\
\hline (7) & (2) & .064 & .034 & .019 & 1.90 & 1.76 \\
\hline (7) & (4) & .081 & .079 & .066 & 1.03 & 1.19 \\
\hline (7) & (5) & .038 & .037 & .026 & 1.04 & 1.40 \\
\hline
\end{tabular}

TABLE 2. Space averages 


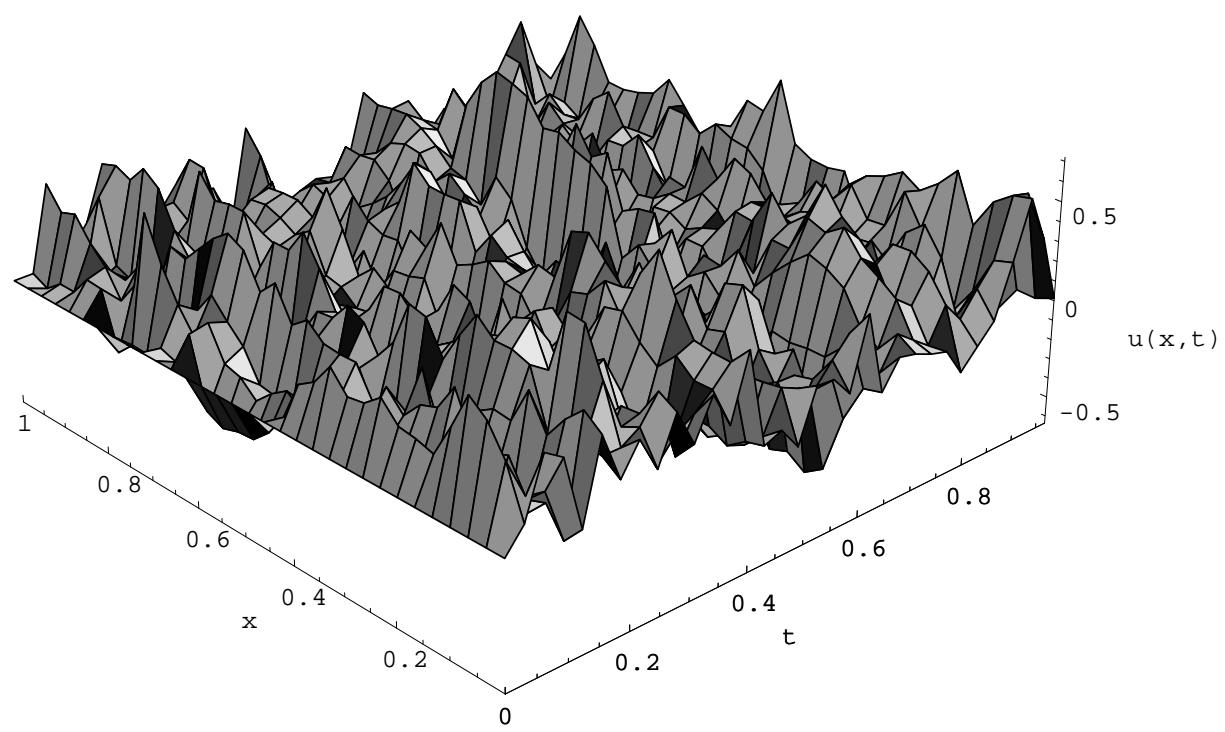

FIGURE 1. Exact solution of the equation with additive noise

4.3. Exact solution. The picture in Figure 1 shows a pathwise solution to equation (3) produced using the method for exact solution described in subsection 2.3 Starting with $u(\cdot, 0)=0$, we chose a final time of $T=1$ and generated the solution on a 32 by 32 grid.

\section{REFERENCES}

1. J. G. Gaines, Numerical experiments with $S(P) D E$ 's, Stochastic Partial Differential Equations (Cambridge) (A. M. Etheridge, ed.), London Mathematical Society Lecture Note Series 216, Cambridge University Press, 1995, pp. 55-71. MR 96k:60154

2. J. G. Gaines and T. J. Lyons, Random generation of stochastic area integrals, SIAM J. on Applied Math. 54 (1994), no. 4, 1132-1146. MR 95f:60063

3. I. Gyöngy, Lattice approximations for stochastic quasi-linear parabolic partial differential equations driven by space-time white noise II, Potential Anal. 11 (1999), 1-37. CMP 99:15

4. N. Ikeda and S. Watanabe, Stochastic differential equations and diffusion processes, North Holland, 1989. MR 90m:60069

Department of Mathematics and Statistics, University of Edinburgh

E-mail address: sandy@ed.ac.uk

Department of Mathematics and Statistics, University of Edinburgh

E-mail address: jessica@ed.ac.uk 